SCIREA Journal of Chemical Engineering http://www.scirea.org/journal/Chemical

May 24, 2021

SCIREA

Volume 4, Issue 1, February 2021

\title{
Enhancing the processes of thermochemical treatment of oil
}

\author{
Ab.H. Rzayev ${ }^{1}$, R.Sh. Asadova ${ }^{1}$, Gurbanov Z.G. ${ }^{2}$ \\ ${ }^{1}$ Institute of Control Systems, Azerbaijan National Academy of Sciences, Baku, Azerbaijan \\ ${ }^{2}$ Baku Higher Oil School, Baku, Azerbaijan \\ Email: Abbas_r@mail.ru, Renaasadova2007@rambler.ru, zafer_adna@mail.ru
}

\begin{abstract}
A systematic analysis of the state of the art in the methods for enhancing processes of thermochemical treatment of oil is carried out. A new method and a new system for controlling the process of dynamic settling of oil emulsion (OE) is developed, which allows increasing the efficiency of managing the process of dynamic settling by more accurately measuring the degree of phase separation, while avoiding the process of "flooding". The mechanism of formation of an electrical double layer around emulsified water droplets (EWD) and the interaction energy of these droplets as a distance function is shown. An adequate mathematical model of hindered settling of EWD is proposed. It is shown that OE and IEL can be broken down by using microwave radiation.
\end{abstract}

Keywords: Oil emulsion,Settler,Water cushion, Intermediate emulsion layer, Dynamic settling 


\section{Introduction}

Treatment of oil in oilfields occupies an intermediate position among the main processes associated with the extraction, gathering and transportation of commercial oil to the consumer (refineries or for export). The efficiency and reliability of the main pipeline transport and the price of commercial oil depend on how the oil is treated in the extraction area. In particular, the content of water and aqueous solutions of mineral salts in oil results in increased transportation costs, causes the formation of stable oil emulsions (OE), creates difficulties in oil refining due to accelerated development of equipment corrosion and catalyst poisoning in the oil cracking process.

It has been established practically that oil demulsification without the use of heat and surfactants (demulsifiers) is not particularly effective. Therefore, currently, about $80 \%$ of all oil extracted with water is treated at thermochemical plants. The difficulty of oil treatment is due to the fact that reservation shells that are adsorption layers of polar oil components, which consist of asphaltenes, naphthenates, microcrystals of refractory paraffins, solid particles of mineral and carbonaceous suspensions, metal porphyrin complexes that give OE high stability, form on the surface of emulsified water droplets (EWD) [1- 4]. And heat and chemical agents (demulsifiers) are used in the thermochemical treatment plant (TCTP) to break the reservation shells (RS).

The enhancement of the oil treatment process (dehydration and desalting) requires a complete breakdown of these RS before the settlers, which work in a dynamic mode, leading to the coalescence and settling of EWD [4]. However, in the field environment, a complete breakdown of RS of EWD is impossible by means of thermochemical treatment of OE alone. As a result, an intermediate emulsion layer (IEL) forms in the settler of TCTP, which consists of concentrated EWD (water content of up to 70\%) with a high content of heavy surfactants (mainly asphaltenes) of oil. In this case, the IEL concentrates on the oil-water interface (hanging beard) [5-9], excessive increase of which can lead to the "flooding" of the settler.

\section{Problem statement}

Our studies have revealed that the enhancement of TCTP processes is closely linked to the effective management of IEL, which leads to an improvement in the quality of the process of dynamic settling of EWD in the settler, since, as noted in our previous studies [5-9], IEL is a hydraulic filter that contributes to the effectiveness of collisions, coalescence and settling of EWD. 
Therefore, investigating the specific characteristics of IEL, identifying control actions that lead to an increase in the effectiveness of IEL and enhancing TCTP processes as a whole is a relevant problem, to which this paper is devoted.

\section{Solution}

A system analysis of TCTP processes has identified the following IEL parameters:

- thickness of the layer;

- formation of an electric double layer around EWD of OE;

- collision frequency of the droplets;

- hindered settling of EWD.

\section{Thickness of IEL}

The value of this parameter is characterized by the content of asphaltenes in oil (optical oil density) and aggregate stability of OE. Due to the importance of this parameter, we propose in this paper a new method and a new system for automatic measurement of the level of water cushion (WC) and the thickness of IEL in the settler that operates in a dynamic mode (Fig.1).

The essence of the proposed method is illustrated by Fig.1. Fig.1, $a$ shows the histogram of the change in the concentration of asphaltenes along the height of the settler, where: $h_{W C}$ is the level of $\mathrm{WC} ; h_{I E L}^{k}$ and $h_{I E L}^{T}$ are the thickness of the concentrated and the transition IEL, respectively; $h_{O}$ is the height of the oil layer; $K_{a}$ is the concentration of asphaltenes, $\mathrm{h}$ is the height of the settler. Fig. $1, b$ shows the diagram of automatic measurement of the degree of phase separation, which includes: 1 - OE supply line into the settler; 2 - settler (can be any shape); 3 - settled water drainage line; 4 - WC; 5 - IEL; 6 - oil layer; 7 - dehydrated oil discharge line; 8 - infra-red radiator (IRR) - $J_{0} ; 8_{1}, 8_{2}, 8_{3}, 8_{4}, 8_{5}, 8_{6}$ - IRR sensors; $8_{1}^{\prime}, 8_{2}^{\prime}, 8_{3}^{\prime}, 8_{4}^{\prime}, 8_{5}^{\prime}, 8_{6}^{\prime}-$ receivers $-J_{i}-$ IRR; 9 - signal converter $J_{0} ; 10$ - signal converter $J_{i}$; 11 - registration and indication block (RIB).

The system operates as follows.

Radiation from source 8 through sensors $81,8_{2} \ldots 8_{6}$ installed at equal intervals along the height of the settler goes through the layer of oil emulsion. Part of the radiation energy is absorbed, and the remaining part is fed to the inputs of sensors $8_{1}^{\prime}, 8_{2}^{\prime} \ldots . . .8_{6}^{\prime}$ of the radiation receiver. The signals from the output of the radiation sensors are received in block 10, where 
they are converted into corresponding signals, based on which the actual concentration of asphaltenes in different phases is calculated in RIB - 11, according to the following algorithm:

$$
\begin{gathered}
C_{i}=\frac{0,434 l_{i} K_{\mathrm{la}}}{D_{i}} \\
D_{i}=\lg \frac{J_{0}}{J_{i}} \\
\mathrm{~K}_{l a}=1,36 \mathrm{M}
\end{gathered}
$$

where $D_{i}$ is the optical density of fluid between the $i$-the sensor and the $i^{l}$ receiver; $J_{0}$ and $J_{i}$ are the intensity of incident light (through the sensors $8_{1}, 8_{2}, 8_{3}, 8_{4}, 8_{5}, 8_{6}$ ) and the intensity of the light transmitted through the medium to the receivers $\left(8_{1}^{\prime}, 8_{2}^{\prime}, 8_{3}^{\prime}, 8_{4}^{\prime}, 8_{5}^{\prime}, 8_{6}^{\prime}\right)$, respectively; $l_{i}$ is the distance between the pair of sensors $i$ and receivers $i^{\prime} ; K_{\text {la }}$ is the coefficient of light absorbtion of asphaltenes; $M$ is the molecular weight of asphaltenes.

Then the histogram is built (Fig.1, a), which is used to determine the degree of phase separation (WC-IEL and IEL-oil).

The technical effect of the proposed method and system is to increase the efficiency of managing the settling process, which consists in the high accuracy of measuring the degree of phase separation, allowing one to reliably control the settling process and completely avoid the "flooding" process.

\section{Formation of EDL around EWD}

The stability of OE also largely depends on the electrical charge on the EWD surface. This is due to the fact that when two different phases (oil, water) come in contact, and especially in case of their relative movement on the interface, the transition of electric charges from the phase with a higher value of electric potential to the phase with a smaller value of electric potential occurs. This transition results in the formation of a charge in one phase and an equal but opposite charge in another. These opposite charges, due to mutual attraction, remain on the interface, forming an electric double layer. It is clear from Fig. 2. that some counter-ions do not have a stable connection with the surface and tend to move away from it, forming a diffuse ionic atmosphere around the particle. In addition to the attraction of ions of opposite polarity, the surface charge repels ions of the same polarity. The manifestation of these opposing forces causes the distribution of positive and negative ions, as the diagram shows in Fig. 2, a. For EWD, according to Koehn's rule 

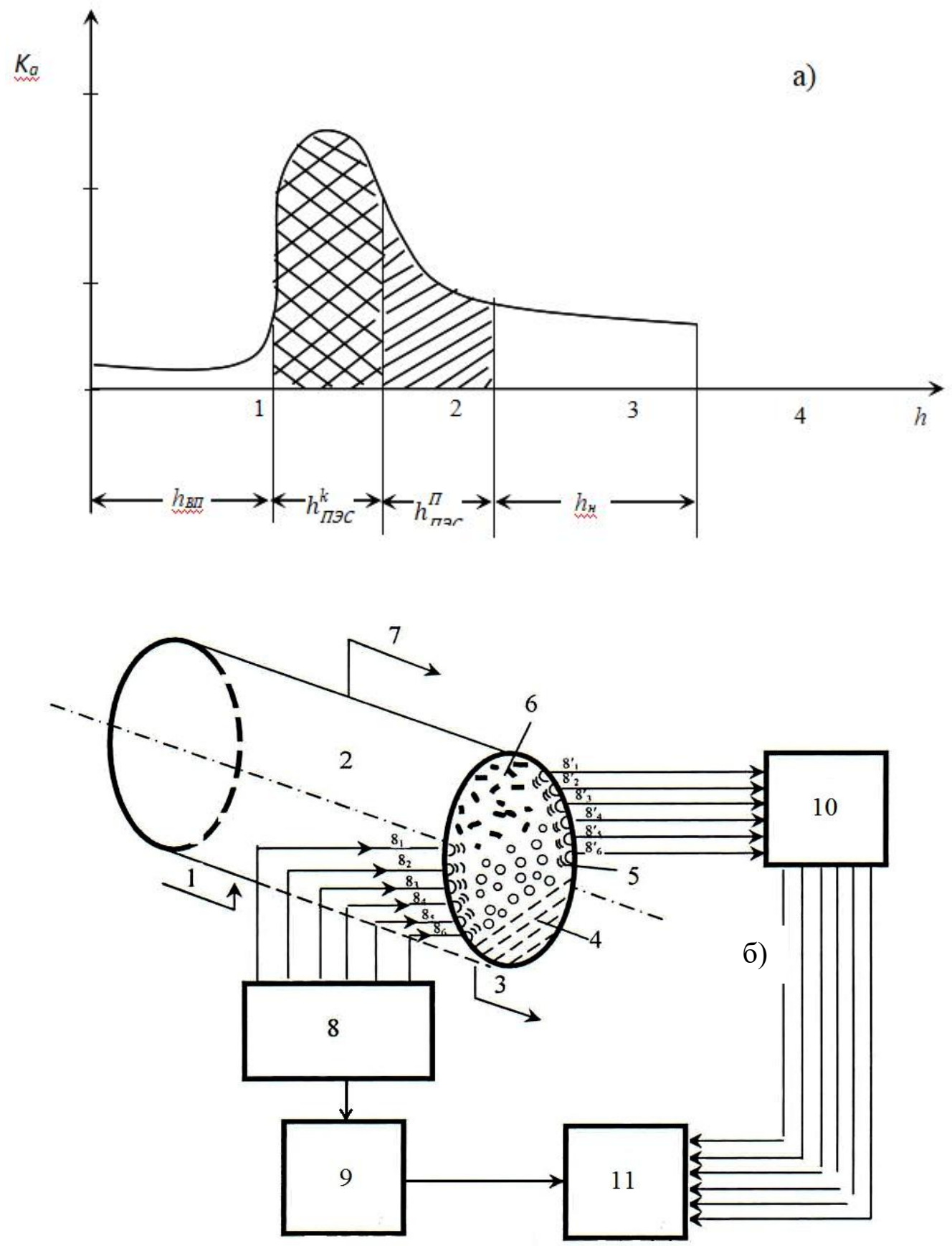

Fig. 1. The method and the system for automatic measurement of the level of water cushion and the thickness of IEL:

a) the histogram of the change in the concentration of asphaltenes along the height of the settler;

b) optical measurement system of the above parameters 
(which states that of two phases in contact, the one with the greater dielectric constant becomes positively charged, i.e. for this reason, substances in contact with water are negatively charged), the surface charge is negative and exchange cations (asphaltenes) act as counterions.

The distribution of ions in the EDL makes the potential change from the maximum on the surface of EWD to zero in the bulk of the oil solution (see Fig. 2, b).
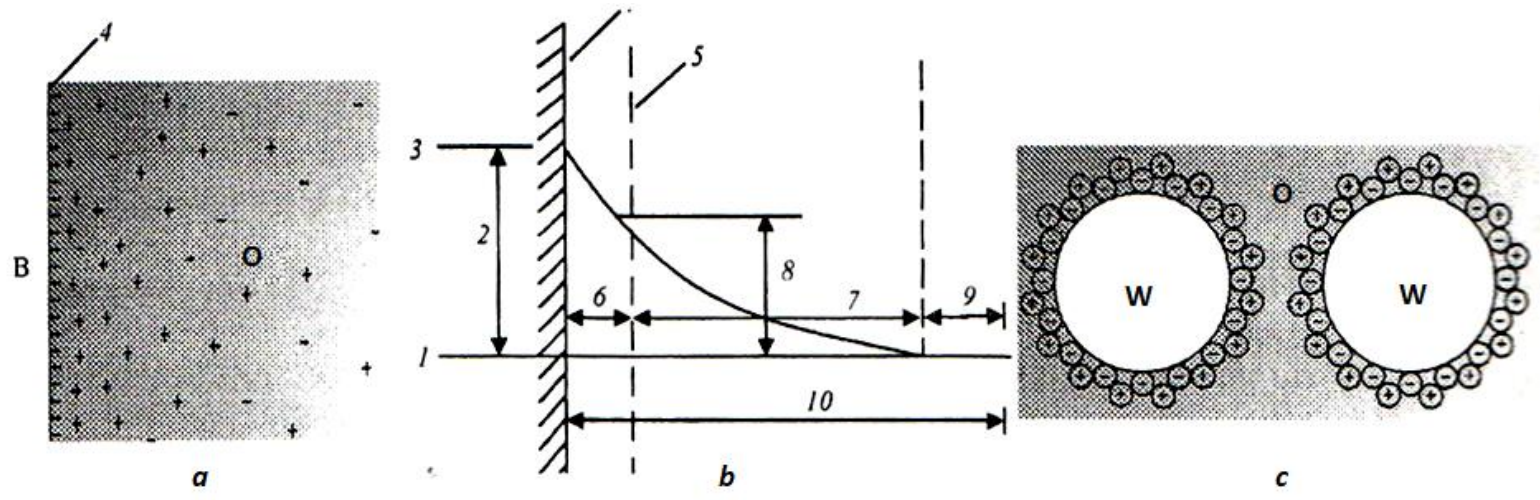

Fig. 2. Electrical double layer on the surface of EWD:

$a$ - model of the diffuse part of the electrical double layer; $b$-diagram illustrating $\zeta$-potential: 1 - fluid potential; 2 - Nernst potential; 3 - potential of the solid; 4 - solid; 5 - shear plane; 6 - adsorption part of ED; 7 - diffuse part of EDL; 8 - $\zeta$-potential; 9 - bulk solution; 10 distance from the solid; $c$ - diagram of mutual repulsion of two EWD with identical surface charges: $\mathrm{W}$ - dispersed phase (water droplet); $\mathrm{O}$ - dispersed phase (oil)

The cationic layer closest to the EWD surface, known as the "Stern layer", is bound to the particle and travels with it, while the ions in the diffuse part of the EDL have independent mobility.

As is clear from Fig. 2, c, when two EWDs with identical surface charges approach each other, a repulsive force emerges, therefore, dispersed EWDs with EDL lead to the stability of OE.

Alongside with the electric repulsion force $\left(U_{e l}\right)$ between the EDL of the same polarity, the long-range Van der Waals-London dispersion force $\left(U_{D}\right)$ also exists between EWD. The stability of $\mathrm{OE}$, in addition to the above factors, also depends on the polarity and magnitude of the interaction, which is the sum of these forces: $U=U_{e l}+U_{D}$.

To determine the force of attraction $U_{D}$ for a pair of identical spheres, Hamaker obtained the expression in the following form [10]: 


$$
U_{D}=\frac{A}{6}\left[\frac{2 r^{2}}{H_{c}^{2}-4 r^{2}}+\frac{2 r^{2}}{H_{c}^{2}}+\ln \frac{H_{c}-4 r^{2}}{H_{c}^{2}}\right],
$$

where $r$ is the radius of the sphere; $H_{c}$ is the distance between the centers of the spheres; $\mathrm{A}$ is the Hamaker-London constant.

Theoretical calculations of A show that its values for liquids range from $1.5 \cdot 10^{-13} \mathrm{erg}$ for oil to $0.5 \cdot 10^{-12}$ erg for water. However, in order to use formula (1) for semi-dispersed colloidal systems, which OE is, it is necessary to take into account the arithmetic mean value of the size (volume) of the pair of EWD. For this purpose, the following formula for the distribution of emulsified water by size proposed by us [11] can be used:

$$
f(r)=\frac{18 W H^{2} \mu_{o}^{2}}{r^{4} T^{2} g^{2}\left(\rho_{w}-\rho_{o}\right)^{2} L^{2}} \cdot \exp \left(-\frac{3 H \mu_{w}}{T g\left(\rho_{w}-\rho_{o}\right) r^{2} L}\right.
$$

where $L$ is the distance between particles; $W$ is volume fraction of water in OE; $\mathrm{H}$ is the height of the level of OE; $\mathrm{T}$ is the time constant; $g$ is the gravity acceleration; $r$ is the radius of EWD. The repulsive forces (energies) of EWD surrounded by EDL can be determined by the Derjaguin-Landau formula [11]:

$$
V_{e l}=\pi \varepsilon \varepsilon_{0} d \psi_{0}^{2} \ln \left[1+e^{-\varkappa H_{0}}\right]
$$

where $H_{0}$ is the distance between spherical surfaces; $\mathcal{\varkappa}$ is the distribution of ions in a diffusion electrical double layer (the reciprocal of the ion cloud radius); $\psi_{0}$ is the particle surface potential; $d$ is the particle diameter; $\varepsilon$ is the relative dielectric permittivity of the outer phase (water at $25^{\circ} \mathrm{C} ; \varepsilon=79$ ); $\varepsilon_{0}$ is the absolute dielectric permittivity $\varepsilon_{0}=8,8542 \cdot 10^{-12} A S / V_{m}$. The superimposition of electrostatic and dispersive interactions is shown in Fig. 3. The values of electrostatic repulsion energy are indicated on the upward ordinate axis, and the values of attraction energy on the downward ordinate axis. The abscissa axis shows the distances between the particles [12-14]. Fig. 3, $b$ shows the secondary minima (potential wells) of the EWD interaction for various values of the surface potentials of EDL. The curve indicated by the dotted line is the dispersion energy of attraction.

In the point $d_{1}$, if the forces of attraction and repulsion between particles decrease the mutual surface potential, then the energy barrier (EB) decreases and the system will undergo slow coagulation. The transition from high stability through slow coagulation to fast one (i.e., to the disappearance of EB) is continuous without a sharp coagulation point. In this point, the particles can join together, forming large agglomerates. However, before they can get close enough to each other, they must overcome the energy barrier (EDL). As the curves show, with 
the ionic strength $J=10^{-3} \mathrm{~mol} \cdot \mathrm{l}^{-1}$ and the Hamaker constant $\mathrm{A}=5 \cdot 10^{-13} \mathrm{erg}$ [14], the value of the surface potential $\varphi_{0} 20 \mathrm{mV}$ is already sufficient to prevent complete coagulation. According to the research results, when the distance between the droplets is less than $50 \mathrm{~nm}$, the rapid thinning and rupture of the interfacial film and the fusion (coalescence) of the droplets begin.

In OE, unlike suspensions, during the interaction of EWD, two factors should be considered, namely: the possibility of distortion of the droplets during their interaction and the presence of a diffuse layer inside the droplets themselves. If the droplets stabilize due to the repulsion of double layers, then a close approach (due to turbulence and OE flow temperature) leads to the flattening of the surfaces; the potential EB counteracting the contact of the droplets (wedging pressure) will be greater than the estimated EB of undeformed spheres. At the same time, the effective radius of curvature increases. The effect of the inner diffusion layer consists in the neutralization of a portion of the surface charge by inner counter-ions, leading to a decrease in the surface potential. Therefore, for this reason, the effect of EDL in a water-in-oil emulsion will be relatively weaker than that in an oil-in-water emulsion.
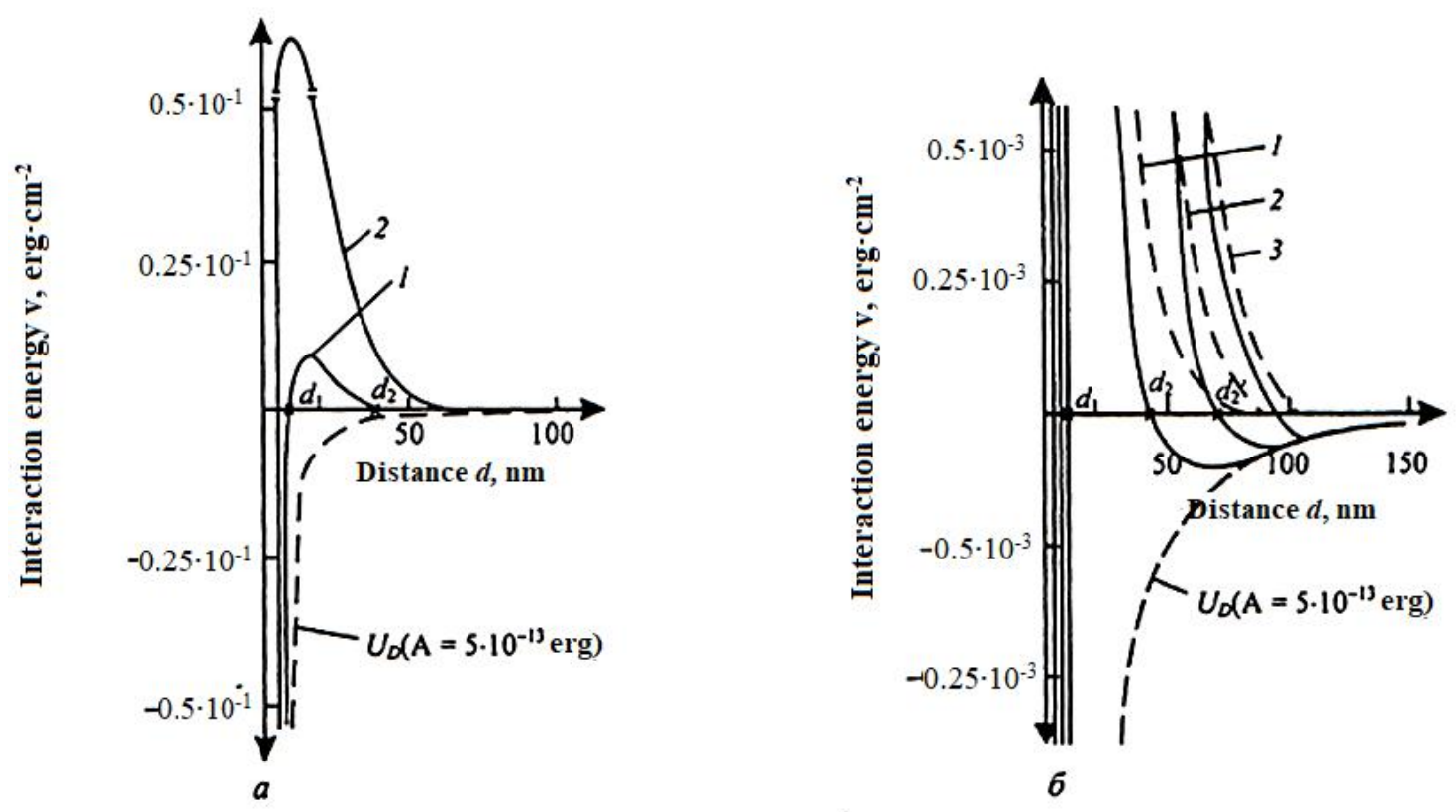

Fig. 3. The interaction energy as a distance function at:

$a-\mathrm{A}=5 \cdot 10^{-13} \mathrm{erg}$ and $J=10^{-3} \mathrm{~mol} \cdot \mathrm{l}^{-1}$ and different surface potential: $1-\psi_{0}=20 \mathrm{mV} ; 2-50$ $\mathrm{mV}$;

$b-\mathrm{A}=5 \cdot 10^{-13} \mathrm{erg}$ (to visualize secondary minima): $1-\psi_{0}=20 \mathrm{mV} ; 2-50 \mathrm{mV} ; 3-200 \mathrm{mV}$

As can be seen from Fig. 3, c, EWD interactions occur at nanoscale distances. Besides the 
EDL theory, there is also the solvation theory that explains the relationship between the high aggregate stability of $\mathrm{OE}$ and the formation of a sufficiently powerful layer of DL molecules on the EWD surface, which prevents particle fusion during collision; and the theory of the structural-mechanical barrier, according to which the stability of OE is determined by the formation of adsorption layers with high structural viscosity on the EWD surface.

\section{Hindered settling of EWD}

The settling of EWD in a concentrated (intermediate layer) occurs in constrained conditions, where, in addition to the viscous friction of the medium (oil), numerous environmental droplets significantly affect water droplets. At the same time, the settling process does not comply with the Stokes formula for determining the settling velocity of a single solid particle. To compare the mathematical description of the process of hindered settling, various formulas were proposed, in particular, Tema's, Maude-Witmer's and Happel's formulas [13].

In [13], the calculations using the formulas of these authors at $W>0.05$ showed conservative values of the settling rate as compared to the experimental data. In view of the above, based on the equation in [13], we have obtained the equation that adequately describes the velocity of hindered settling of droplets from a concentrated layer:

$$
\vartheta_{k}=1,5 \vartheta_{c \mathrm{~T}}(1-W)^{5 / 2} \cdot \frac{\mu_{\varphi}-\left(3 \mu_{\varphi}-\mu_{c}\right)(1+K)^{2}+2\left(\mu_{\varphi}-\mu_{c}\right)(1+K)^{3}}{\left(3 \mu_{\varphi}+2 \mu_{c}\right)(1+K)^{3}}
$$

where $K=\sqrt[3]{\frac{6,2}{8 W}}, \vartheta_{c}=\frac{2}{9} \cdot \frac{\Delta \rho g r^{2}}{\mu_{c}}$ is the Stokes equation for the settling rate of a single solid particle, which is also applicable to calculating the settling rate of a set of particles if $W \leq$ 0,05 and $r<\sqrt{\sigma /(g \Delta \rho)}$

In deriving equations (2), the following assumptions were made:

a) the flow around particles is viscous, proceeding from the condition of smallness of the number $R_{e}=\left|U_{g}\right| \frac{2 r}{v} \ll 1$, and it is described in the first approximation by the Stokes linear law $\left(F_{5}=6 \pi \mu_{\varphi} r \vartheta_{c \mathrm{~T}}, \vartheta_{c \mathrm{~T}}=\vartheta-\vartheta_{\Pi}\right)$

b) the droplets are strictly spherical in shape. In the case of their deformability, the form factor should be introduced, and in the case of their polydispersity, they should be considered fractionally;

c) the average distance between particles with an ordered arrangement is determined from the 
formula $l=2 r \cdot \sqrt[3]{\frac{6,2}{8 W}}$;

d) the movement of the droplets is not affected by the forces that cause their transport due to turbulent and upward migrations, as well as electrostatic, thermophoretic, diffusiophoretic and other non-hydrodynamic forces, with the exception of gravity which causes the particles to settle;

e) on the surface of the droplet, the nonslip condition is satisfied, i.e. the velocity of the fluid should be equal to the average velocity of the droplets;

f) at the distance $\alpha \rightarrow r+l_{k} / 2$, the determination of velocity in a liquid medium obeys the condition

$$
\vartheta_{r_{c}}=\vartheta_{k}\left(\frac{1}{1-W}\right)^{n} \cos \theta_{c}, \vartheta_{\theta_{c}}=-\vartheta_{k}\left(\frac{1}{1-W}\right)^{n} \sin \theta_{c}, \quad l_{k}=l-2 r
$$

$\vartheta_{r_{c}}, \vartheta_{\theta_{c}}$ are the normal and tangential components of velocity.

\section{Methods of enhancing the process of $\mathrm{OE}$ demulsification}

Methods based on microwave radiation have been widely used at oil refineries in recent years for the breaking of heavy oil fractions, demulsification of OE, viscosity reduction, asphaltene content and enrichment of the vacuum residue [14-17]. The advantage of these methods, compared to conventional thermal breakdown, is that two phenomena occur simultaneously during microwave radiation. The first phenomenon is due to an increase in temperature that leads to a decrease in viscosity and coagulation, as a result of which EWD are separated from oil without the use of a chemical agent. The second phenomenon is coagulation. 


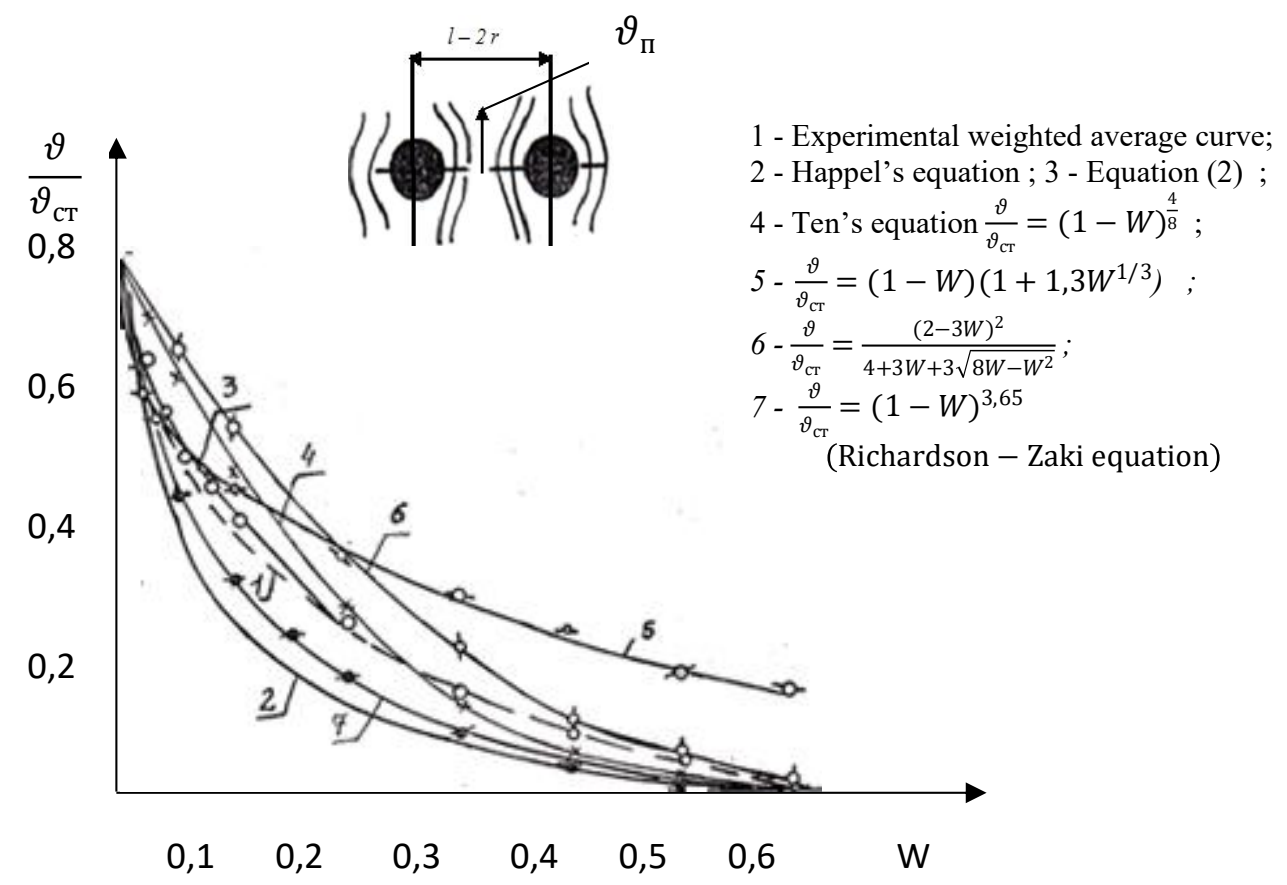

Fig. 4. Dependence of the velocity of hindered settling on the volume fraction of droplets

High temperature and low viscosity makes the coagulation process easier. Besides, during microwave radiation, EWD rotate and the zeta ( ) potential decreases (see Fig. 2), which leads to the enhancement of the process of dynamic settling. To increase the efficiency of the dynamic settling of OE in the settler of TCTP, the system developed for controlling and managing the processes of thermochemical oil treatment can be used along with microwave radiation. The system allows creating artificial oscillatory motion (compression and expansion) of IEL in the settler [18] and therefore increasing the efficiency of the dynamic settling of OE.

\section{Conclusions}

A new method, an algorithm and a system for automatic measurement of the water cushion level and the thickness of the intermediate emulsion layer in settlers based on measuring the optical density of oil are proposed. It is shown that the main obstacle to enhancing the process of coalescence and dynamic settling of oil emulsion is the electrical double layer on the surfaces of emulsified water droplets (EWD). The characteristics of the EWD interaction and the estimates of the forces of attraction and repulsion of these droplets are given. A formula for the distribution of emulsified water by size is also proposed. 


\section{References}

[1] S.G. Lutoshkin, Gathering and treatment of oil, gas and water for transport (in Russian), M.: Nedra, 1972, 324 p.

[2] G.N. Pozdnyshev, Stabilizing and breaking oil emulsions (in Russian), M.: Nedra, 1982, $221 \mathrm{p}$.

[3] I.L. Markhasin, Physical and chemical mechanics of oil reservoir (in Russian), Moscow, Nedra, 1977, $214 \mathrm{p}$.

[4] Ab.G. Rzayev, G.I. Kelbaliyev, G.I. Mustafayeva, S.R. Rasulov, Simulation of the formation and breakdown of emulsion during thermochemical treatment of oil (in Russian). Chemistry and Technology of Fuels and Oils, Moscow, 2018, No.3, pp. 11-18.

[5] Innovation. Microwave method of breaking oil emulsions (in Russian). Oil and Gas Technologies, Moscow, No1, 2001, pp.107-108.

[6] T.A. Aliyev, Ab.G. Rzayev, G.A. Guluyev, S.F. Babayev, I.A. Nuriyeva, Eurasian patent No 026554, 28.04.2017, Method and system for controlling the process of dynamic settling of oil emulsion,

[7] T.A. Aliyev, Ab.G. Rzayev, G.A. Guluyev, G.I. Kelbaliyev, I.A. Nuriyeva, Eurasian patent No 029244, 28.02.2018, Method and system for controlling the process of thermochemical dehydration of oil emulsion,

[8] Ab.G. Rzayev, The scientific foundations for calculating the design and management of processes of separation of oil emulsion in oil treatment and oil refining (in Russian). Dissertation for the degree of $\mathrm{PhD}$ in Engineering, Baku, Institute of Theoretical Problems of Chemical Technology, 1994

[9] Ab.G. Rzayev, S.R. Rasulov, I.A. Abbasova, S.N. Ragimova, Development of the system for controlling the process of dynamic settling of oil emulsion (in Russian). Equipment and Technologies for Oil and Gas Complex, Moscow, 2014, No 5, pp.40-43

[10] T.A. Aliev, G.A. Guluyev, Ab.G. Rzayev, Mathematical models of the intermediate emulsion layer in the settlers of the thermochemical oil treatment plant (in Russian). Oil Refining and Petrochemistry, Moscow, 2011, No.8, pp.50-53

[11] Emulsion science. Ed. Philip Sherman. Academic Press, London, 1968, 496 p.

[12] Ab.G. Rzayev, I.A. Nurieva, S.R. Rasulov, Investigation of the mechanisms of coalescence of dispersed phase droplets and separation of oil emulsions (in Russian). Equipment and Technologies for Oil and Gas Complex, Moscow, 2016, No 4, pp.64-67

[13] G.I. Kelbaliev, A.H. Rzaev, A.F. Guseinov, A.A. Kasumov, Sedimentation of the 
particles from a concentrated dispersed flox. Plenum publishing corporation. 00220841/91/6193-10645/ 1250C, 1992, c.365-368

[14] Adango Miandonye, Brittany Macdonald, Microware radiation induced Visbreaking of Heavy Crude Oil Journal of Petroleum Science Research (JPSR), Valume 3, Issu 3, 2014 , pp .130-135.

[15] H. Abdurahman, Nour and Rosli, M. Yunus, A comparative study on emulsion demulsification by microware radiation and conventional heating. http/scialert.net/fulltext/?da=jas.2006,2307,2311

[16] Zarrin Nasri, Application of microware technology in asphaltene and viscosity reduction of iranian vacuum residue. Proseedings of Academicsera $3^{\text {rd }}$ International Conference, Jacarta, Indonezia, 13-14 March, 2017, ISBN, 978-93-86083-39-0, pp. 46-49.

[17] Ab.G. Rzayev, S.N. Ragimova, S.R. Rasulov, I.A. Abasova, Developing a system for controlling and managing the processes of thermochemical treatment of oil (in Russian). Automation, Telemechanization and Communication in Oil Industry, Moscow, 2013, No 12, pp.38-41 